\title{
Influence of meditation on anti-correlated networks in the brain
}

\author{
Zoran Josipovic $^{1}{ }^{*}$, Ilan Dinstein ${ }^{2}$, Jochen Weber $^{3}$ and David J. Heeger ${ }^{1}$ \\ ${ }^{1}$ Department of Psychology and Center for Neural Science, New York University, New York, NY, USA \\ 2 Neurobiology Department, Weizmann Institute of Science, Rehovot, Israel \\ ${ }^{3}$ Department of Psychology and SCAN, Columbia University, New York, USA
}

\section{Edited by:}

Amishi P. Jha, University of Miami, USA

Reviewed by:

Oliver C. Schultheiss, Friedrich

Alexander University, Germany Chien-Te Wu, National Taiwan

University College of Medicine, Taiwan

*Correspondence:

Zoran Josipovic, Department of Psychology and Center for Neural Science, New York University, Room 957, 6 Washington Place, New York, NY 10003, USA.

e-mail:zoran@nyu.edu
Human experiences can be broadly divided into those that are external and related to interaction with the environment, and experiences that are internal and self-related. The cerebral cortex appears to be divided into two corresponding systems: an "extrinsic" system composed of brain areas that respond more to external stimuli and tasks and an "intrinsic" system composed of brain areas that respond less to external stimuli and tasks. These two broad brain systems seem to compete with each other, such that their activity levels over time is usually anti-correlated, even when subjects are "at rest" and not performing any task. This study used meditation as an experimental manipulation to test whether this competition (anti-correlation) can be modulated by cognitive strategy. Participants either fixated without meditation (fixation), or engaged in non-dual awareness (NDA) or focused attention (FA) meditations. We computed inter-area correlations ("functional connectivity") between pairs of brain regions within each system, and between the entire extrinsic and intrinsic systems. Anti-correlation between extrinsic vs. intrinsic systems was stronger during FA meditation and weaker during NDA meditation in comparison to fixation (without mediation). However, correlation between areas within each system did not change across conditions. These results suggest that the anti-correlation found between extrinsic and intrinsic systems is not an immutable property of brain organization and that practicing different forms of meditation can modulate this gross functional organization in profoundly different ways.

Keywords: brain networks, functional connectivity, default mode, intrinsic system, extrinsic system, meditation, non-dual awareness, mindfulness

\section{INTRODUCTION}

The cortex is hypothesized to be divided into two broad systems. One responds positively to tasks involving external stimuli and includes the sensory and motor systems. The other responds more strongly when not engaged in a task and includes less frequently studied brain areas such as the precuneus, the inferior parietal lobule, and medial prefrontal cortex (mPFC). The first system has been named the extrinsic or task-positive system while the second system has been named the intrinsic, task-negative, or default system (Gusnard and Raichle, 2001; Fox et al., 2005; Fransson, 2006; Fox and Raichle, 2007; Golland et al., 2007, 2008; Tian et al., 2007; Buckner et al., 2008; Boly et al., 2009; Soddu et al., 2009; Wang et al., 2010; Vanhaudenhuyse et al., 2010a). An intriguing finding is that spontaneous activity over time in these broad systems tends to be anti-correlated even during rest and sleep, when no stimulus is being presented and no task is being performed (Fox et al., 2005; Fukunaga et al., 2006). The functional significance of this anti-correlation is not understood, but has been interpreted as evidence for an antagonistic or competitive relationship between these two cortical systems (Gusnard and Raichle, 2001; Fox et al., 2005; Raichle and Snyder, 2007).

These findings have led to a flurry of suggestions and speculations regarding the functional significance of the intrinsic system.
Some of the efforts to elucidate the functional role of intrinsic system areas have focused on finding "internally oriented" tasks that evoke positive responses in intrinsic areas. In one example of such a study, different intrinsic system areas exhibited positive fMRI responses during tasks that involved making decisions about one's current personal state, constructing a scene from memory, or imagining the future (Andrews-Hanna et al., 2010). Other examples have included concentrating on "volitional-prospection" thoughts (e.g., what will you do to advance your research project?) (Preminger et al., 2011), and simulating problem-solving (e.g., you're stuck with someone else's ring on your finger; simulate what you would do to take it off) (Gerlach et al., 2011). These tasks also generated sustained positive fMRI responses in intrinsic system brain areas, which exhibited negative fMRI responses during externally oriented tasks.

A second approach to studying the function of the intrinsic system has been through assessment of inter-area correlations over time (also known as "functional connectivity"), during different tasks and across different patient populations. The extent of inter-area correlation between two brain areas during any given time period is thought to reflect the synchronization of neural activity between the two areas, due to a combination of anatomical connections between the two areas as well as common 
inputs (Friston, 1995). There is an implicit assumption in the field that distinct brain areas that share a function ("work together") should exhibit synchronized changes in activity over time. Interarea correlation may, therefore, be a useful measure for determining the functional integrity/coupling across areas belonging to a distributed brain system. Patients with Alzheimer's disease exhibit weaker inter-area correlations between intrinsic system areas, suggesting a possible role of these areas in introspective memory (Sorg et al., 2007). Individuals with autism also exhibit weaker inter-area correlations between intrinsic system areas, suggesting a possible role of these areas in social behaviors that are abnormal in autism (Kennedy and Courchesne, 2008). Abnormal intrinsic system interactions have also been found in individuals with Attention Deficit Hyperactivity Disorder (ADHD), suggesting that activity in the intrinsic system might interfere with sustained attention and task engagement (Sonuga-Barke and Castellanos, 2007; Castellanos et al., 2008). Other studies have reported that inter-area correlations are larger between intrinsic system areas during hypnosis (Demertzi et al., 2011), and that they exhibit various changes during different stages of sleep (Horovitz et al., 2009) and sedation (Stamatakis et al., 2010), suggesting that an individual's state of awareness can have significant effects on the synchronization between these areas.

A highly speculative but intriguing idea is that the broad segregation of the cortex into extrinsic and intrinsic systems might somehow reflect the segregation of human awareness/consciousness into extrinsic and intrinsic experiences (Golland et al., 2008; Soddu et al., 2009). Asian contemplative philosophies, going back to at least fourth century CE (Conze, 1993), and perhaps much earlier (Radhakrishnan, 1995), have described the structuring of human experience along the subjectobject dichotomy, accompanied by a competition between internally and externally related mental processes. We can all intuitively relate to the feeling of being lost in internal thoughts, when we become less aware of external stimuli. Alternatively, we have all experienced being entirely immersed/engaged in an external task (e.g., watching a movie, writing a paper) during which we completely forget about everything else.

According to this idea, it might be possible to use meditation to voluntarily manipulate the inter-area correlations between the intrinsic and/or extrinsic system brain areas. Meditations form a large body of techniques that enable individuals to influence their state of awareness and enhance their well-being, by attending to various aspects of internal or external experience (Cahn and Polich, 2006; Lutz et al., 2007, 2008). For the purpose of cognitive neuroscience research, different meditation techniques have been classified into three broad categories: focused attention (FA), mindfulness or open monitoring (OM), and non-dual awarness (NDA) (Jha et al., 2007; Lutz et al., 2007, 2008; Austin, 2009; Josipovic, 2010; Travis and Shear, 2010). fMRI studies have reported that participants practicing FA and OM meditations exhibited larger anti-correlations between several areas of extrinsic and intrinsic systems and larger correlations between areas within each system compared to when they were not meditating, but have not looked at the anti-correlation levels between the two systems (Farb et al., 2007; Pagnoni et al., 2008; Brewer et al., 2011; Jang et al., 2011; Kilpatrick et al., 2011; but see Manna et al.,
2010). In addition, one study reported larger correlations between of posterior cingulated cortex (PCC) (generally considered part of the intrinsic system) and anterior cingulate cortex (ACC) (generally considered part of the extrinsic system), for experienced mediators practicing FA and OM meditations and ACC (Brewer et al., 2011). These results have been interpreted as evidence for increased functional segregation between extrinsic and intrinsic systems and increased within system synchronization during these styles of meditation. These results might be interpreted as suggesting that inter-area correlations within and between the intrinsic and extrinsic systems can be controlled voluntarily by choosing a specific cognitive strategy. Before drawing such a conclusion, however, it is critical to establish the specificity of the results. Would similar changes in inter-area correlations occur for any type of meditation, or for any of a number of non-meditation tasks?

In the current study we assessed whether NDA meditation has a similar effect on inter-area correlations within and between intrinsic and extrinsic systems. Experienced meditators from the Tibetan Buddhist tradition participated in three separate fMRI scans during which they fixated without meditation (fixation), engaged in FA meditation, and engaged in NDA meditation. This experimental design enabled us to measure inter-area correlations within the same subjects during three different mental states. In agreement with previous studies, we found that the anticorrelations between intrinsic and extrinsic systems were larger during FA meditation than during fixation. In contrast, the anticorrelations between the two systems were smaller during NDA meditation. The specificity of these results strongly supports the conclusion that inter-area correlations between the intrinsic and extrinsic systems can be controlled voluntarily. The results add to the growing body of scientific evidence that mental states induced by different meditation practices correspond to measurably different states of brain activity. Furthermore, the results support the intuitive, but speculative, idea that the typical anti-correlation between the extrinsic and intrinsic systems might reflect the duality of extrinsic and intrinsic experiences, and that NDA meditation enables an atypical state of mind in which extrinsic and intrinsic experiences are increasingly synergistic rather than competing.

\section{METHODS \\ PARTICIPANTS}

Twenty-four participants, experienced in the Tibetan Buddhist meditation practices of NDA and/or FA, participated in the experiments (16 male, eight female; median age, 50 years, range 32-62 years, all but one right-handed; Caucasian, 19, Asian four, Hispanic one). The data from two of the participants were discarded because they fell asleep during the experiment. Of the remaining 22 participants, 14 participated in both meditations, four participated only in NDA, and four only in FA. Participants were selected, on the basis of recommendations from their teachers, from various Buddhist meditation centers in the US. Their experience ranged from 8 to 33 years of meditation; this corresponds roughly to $4000-37,000 \mathrm{~h}$ of practice, (mean $13,727 \pm$ 8920 STD), based on participant's reports of their daily practice, and assuming an average of $10 \mathrm{~h}$ per day for retreat practice 
(8-14 h/per day in most Tibetan Buddhist traditions). While we strived for homogeneity, and most of our practitioners (17), had experience only with the Tibetan Buddhism, seven had some experience with other contemplative traditions as well, such as Mindfulness (1), Vedanta (2), Taoism (3), and Zen (1). All participants had normal or corrected-to-normal vision, provided written informed consent, and were paid for their participation in the study. Experiments were conducted in accordance with the safety guidelines for fMRI research, as approved by the University Committee on Activities Involving Human Subjects at New York University.

\section{INSTRUCTIONS TO PARTICIPANTS}

Instructions to participants for "fixation" were: "Keep your gaze on the fixation point and allow your mind to wander naturally, and avoid engaging in any type of meditation." For viewing of movie clips the instruction was the same, except without fixating. FA meditation is common to all schools of Buddhism and, in one form or another, to all other contemplative traditions. Thus, the instructions were standard: "Keep a steady focus on the object of meditation, the fixation point, to the exclusion of other mental content, and if thoughts arise do not follow them but remain focused on the fixation point. If your mind wanders off, bring it back gently to the object of focus." For NDA meditation the instructions were: "Rest in reflexive NDA, equally aware of inside and outside of your body, allowing experiences to arise and subside of their own accord." NDA meditators were also told to avoid common pitfalls in this style of meditation, such as, fixating attention either exclusively on the external or exclusively on the internal experience or, trying to keep one's mind empty of thoughts and feelings through holding effortful focus.

We did not have a reliable method of assessing the quality of meditation experience in the scanner or the effectiveness with which subjects were able to enter meditative states, beyond the most rudimentary, i.e., whether subjects fell asleep or not (eyetracker), or whether they were able to meditate at all (self-report after each run). However, the possibility that their meditation was of a poor quality would not present a confound for our results, but rather would have diminished any differences in brain activity between conditions.

\section{VISUAL STIMULI}

Stimuli were presented via an LCD projector and custom optics onto a rear-projection screen in the bore of the MRI scanner. Participants were supine and viewed the projected stimuli through an angled mirror. An infrared, video eye-tracker (ASL RLRO-6, Bedford, MA, or EyeLink 1000, SR Research, Mississauga, ON, Canada) was used to visually monitor whether fixation was maintained and if the eyes were kept open, but the eye-position measurements were not recorded.

\section{PROTOCOL}

Each MRI scanning session consisted of four scans. Each of the first three were $480 \mathrm{~s}$ in duration, during which participants fixated a red dot located in the center of the screen on a neutral gray background. The participants either fixated (fixation), or engaging in NDA, or FA meditations (see Discussion for an explanation of differences between NDA, FA, and fixation). The order of these first three scans was counterbalanced across participants. During the fourth scan, participants viewed movie clips (10 s each), alternating with blank periods of neutral gray ( $8 \mathrm{~s}$ each). The movie clips showed faces, urban buildings, natural outdoor scenes, and various objects, and were presented in randomly shuffled order (Hasson et al., 2003). The experiment began and ended with neutral gray (20s), and lasted for a total of $608 \mathrm{~s}$ per scan. For the meditation scans, participants were given time to enter into meditation before $\mathrm{fMRI}$ acquisition commenced. This way we avoided confounding the neural correlates of NDA or FA with the neural correlates of techniques to get to these states.

\section{MRI ACQUISITION}

MR images were acquired at the NYU Center for Brain Imaging, using a Siemens Allegra 3T head-only scanner equipped with a head coil (NM-011, Nova Medical, Wakefield, MA). Blood oxygenation level-dependent (BOLD) contrast was obtained using a T2*-sensitive echo planar imaging (EPI) pulse sequence $(\mathrm{TR}=2000 \mathrm{~ms}, \mathrm{TE}=30 \mathrm{~ms}, 36$ axial slices, $3 \times 3 \times 3 \mathrm{~mm}$, $64 \times 64$ matrix in a $192 \times 192 \mathrm{~mm}$ FOV). In addition to functional images, a high-resolution, T1-weighted anatomical volume of images was acquired for each participant, using an MPRAGE sequence $(\mathrm{TR}=2500 \mathrm{~ms}, \mathrm{TE}=3.93 \mathrm{~ms}, \mathrm{TI}=900 \mathrm{~ms}$, flip angle $=$ $8^{\circ}, 176$ sagittal slices, $1 \times 1 \times 1 \mathrm{~mm}, 256 \times 256$ matrix in a $256 \mathrm{~mm}$ FOV).

\section{PRE-PROCESSING AND SEGMENTATION}

fMRI data were processed using standard tools available in the Brain-Voyager QX 2.2 software package (Brain Innovation, Maastricht, The Netherlands) (Goebel et al., 2006), with NeuroElf (http://neuroelf.net/, JW), with custom software written in Matlab (MathWorks, Natick, MA), and with Excel (Microsoft, Seattle, WA). The first four volumes of each fMRI scan were discarded, to allow the magnetization to reach steady state, and to allow participants time to recover from the onset of scanner noise. Functional MRI time-series were pre-processed with slice-time correction (to compensate for the slightly different times during which each slice was acquired), 3D motion correction (which involved re-sampling with windowed sinc interpolation), and temporal high-pass filtering (with a cutoff frequency of $0.006 \mathrm{~Hz}$, removing trends with periods greater or equal to $150 \mathrm{~s})$. The fMRI time-series were then corrected for serial correlation (Goebel et al., 2006), and temporally smoothed using a Gaussian kernel with a temporal extent of $8 \mathrm{~s}$ (four TRs, full width at half maximum). Anatomical images were corrected for spatial inhomogeneity in intensity, and transformed, using trilinear interpolation, to the Talairach coordinate system (Talairach and Tornoux, 1988). Functional images were aligned with the high-resolution anatomical volume using trilinear interpolation. Other than the interpolation during these transformations, no additional spatial smoothing was performed.

Artifacts due to non-neural signals such as head-motion, breathing, and heart rate were minimized as follows. The head motion variables were projected out (using linear regression) from the fMRI time-series. A pair of global components were extracted and removed: (1) the mean fMRI response time-series 
for all cortical gray matter voxels, and (2) the mean fMRI response time-series for all white matter and subcortical voxels. The cortical surface was reconstructed from the high-resolution anatomical images, separately for each participant, to identify the gray and white matter voxels. These global components were removed by projecting them out of the response time-series for each individual voxel (Golland et al., 2007; Fox et al., 2009).

\section{DEFINING EXTRINSIC AND INTRINSIC SYSTEM ROIs}

Fifteen regions of interest (ROIs) were defined separately in each individual participant (Figure 1). Eight of these ROIs were part of the extrinsic system, exhibiting more activity during external stimulation, while seven were a part of the intrinsic system, exhibiting less activity during external stimulation (Fox et al., 2005; Damoiseaux et al., 2006; Fransson, 2006; Goldberg et al., 2006; Nir et al., 2006; Fox and Raichle, 2007; Golland et al., 2007, 2008; Castellanos et al., 2008; Vincent et al., 2008; Vanhaudenhuyse et al., 2010a). The ROIs were defined based on a combination of anatomical and functional criteria using the movie clip scan. Once the ROIs were defined, the main experimental measures (e.g., level of correlation/anti-correlation between these ROIs during NDA, FA, and fixation) were assessed using statistically independent $\mathrm{fMRI}$ data acquired in separate scans.

Brain regions were identified as responding either more strongly to the movie clips than neutral gray (all movie clips $>$

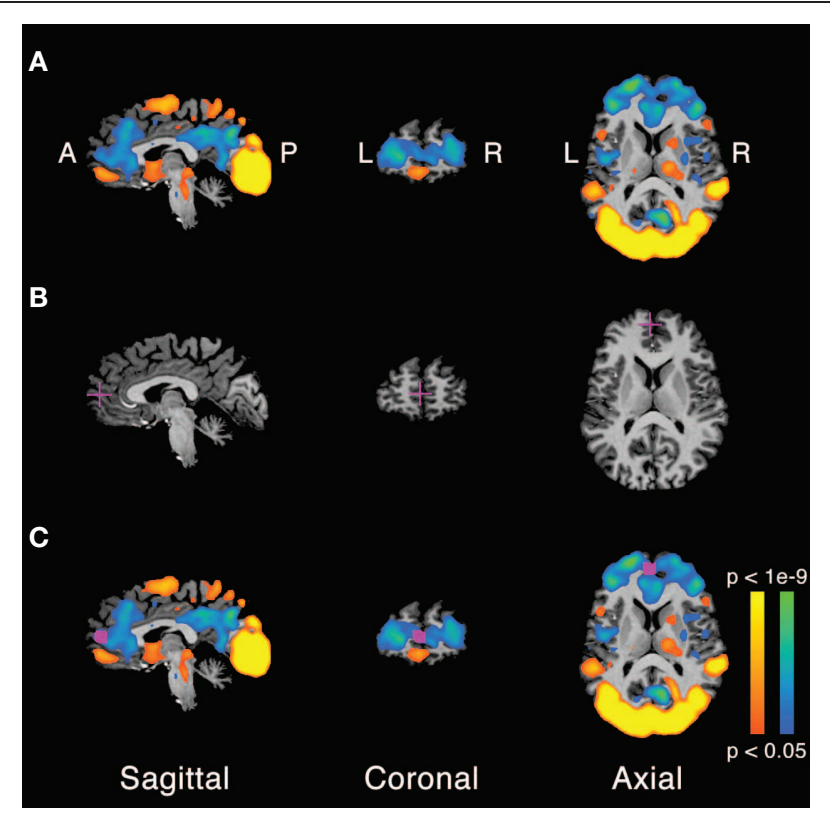

FIGURE 1 | Extrinsic and intrinsic system regions of interest (ROIs).

(A) Brain areas activated by the movie clips, for a typical example participant. Orange/yellow, activity evoked by movie clips $>$ uniform gray screen. Blue/green, movie clips < uniform gray screen. A, anterior. $P$, posterior. $R$, right and $L$, left. (B) Anatomical location of the medial prefrontal cortex (mPFC) ROI in this example participant. Cross-hairs, voxel corresponding to the center of the ROI. (C) Anatomically and functionally defined ROI. Orange/yellow and blue/green, activation copied from panel A. Magenta, voxels exhibiting statistically significant activity (panel A) within a cube (12 $\mathrm{mm}$ on each side) surrounding the center voxel (panel B). neutral gray) or less strongly to the movie clips than neutral gray (all movie clips < neutral gray), following previous reports of segregating the extrinsic and intrinsic networks by examining brain activity evoked by watching movies (Hasson et al., 2003, 2010; Golland et al., 2007). In addition, area Lateral Occipital (LO) was defined by identifying brain regions that responded more strongly to movie clips of objects than all other movie clips (Hasson et al., 2003). We used Brain Voyager QX to run a standard statistical parametric mapping (SPM) analysis (Friston et al., 1994). In short, we constructed a general linear model (GLM) for the underlying neural responses to each movie clip. The regression matrix contained one row for each time point, in which neural activity was modeled as either "on" $=1$ or "off" $=0$, and five columns, one for each type of movie clip (visual motion, faces, objects, and houses) as well as a column of ones for the mean image intensity. The periods of neutral gray between movie clips served as the baseline. The columns of this regression matrix were convolved with a canonical hemodynamic impulse response function (HIRF) to create a model of the expected fMRI response time-series (Boynton et al., 1996; Glover, 1999). We used linear regression to estimate response amplitudes (beta values) for each voxel and each condition, solving an equation of the form $y=$ $A x+\varepsilon$, where the vector $y$ was the measured fMRI time-series during one scan, the vector $x$ contained the estimated response amplitudes, $A$ was the regression matrix described above, and $\varepsilon$ was the residual variance, orthogonal to the model $A$. Finally, voxels exhibiting statistically significant response differences were identified (Figure 1A; $p<0.05$; $t$-test, not corrected for multiple comparisons across voxels, but with a minimum cluster volume of $162 \mathrm{~mm}^{3}$ ).

A set of anatomical landmarks were identified by hand, one for each ROI in each participant's brain, and the functional activation in the vicinity of these landmarks was used to define the ROIs. Specifically, we identified the voxel within $8 \mathrm{~mm}$ of each landmark, separately for each individual participant, which exhibited the strongest functional activation (Figure 1B). This voxel was selected as the ROI center for that ROI in that participant. Each ROI was then created as a cube (12 mm on each side) surrounding the ROI center. Finally, each ROI was restricted to include only voxels exhibiting statistically significant (although not corrected for multiple comparisons) response differences (Figure 1C). Hence, the ROI centers and sizes differed across participants (Table 1).

Eight extrinsic ROIs (four in each hemisphere) were defined (Table 1): "Vis," an early visual area located in and around calcarine sulcus; LO, area in LO and inferior temporal sulcus; V3+, in and around middle occipital gyrus; SPL, superior parietal lobule in and around the superior intra-parietal sulcus. Each ROI was defined separately for each hemisphere.

Seven intrinsic ROIs were defined: (Table 1): PCC, in and around the rostral portion of posterior cingulate gyrus; right and left LP, lateral parietal area in the inferior parietal lobule defined separately in each hemisphere; Rsfg, right superior frontal gyrus (BA8/6); mPFC, perigenu area of mPFC; cPrec, in and around ventral portion of subparietal sulcus and posterior to precuneal sulcus; dPrec, in and around dorsal caudal portion of subparietal sulcus. There were two peaks of de-activation in the precuneus, 
Table 1 | ROI locations and sizes.

\begin{tabular}{lccll}
\hline ROI & $\mathbf{X}(\mathbf{m m})$ & $\mathbf{Y}(\mathbf{m m})$ & $\mathbf{Z}(\mathbf{m m})$ & Vol $\left(\mathbf{m m}^{3}\right)$ \\
\hline I-Vis & $-9 \pm 2.6$ & $-86 \pm 4.2$ & $-7 \pm 4.1$ & $1700 \pm 63$ \\
r-Vis & $7 \pm 2.1$ & $-85 \pm 4.4$ & $-6 \pm 5$ & $1702 \pm 72$ \\
I-LO & $-47 \pm 3.7$ & $-66 \pm 3.4$ & $-5 \pm 3$ & $1540 \pm 290$ \\
r-LO & $44 \pm 5$ & $-63 \pm 5.5$ & $-6 \pm 3.2$ & $1394 \pm 382$ \\
I-V3+ & $-26 \pm 4.8$ & $-86 \pm 2.8$ & $14 \pm 4.2$ & $1672 \pm 99$ \\
r-V3+ & $20 \pm 6$ & $-86 \pm 4$ & $15 \pm 3.8$ & $1625 \pm 125$ \\
I-SPL & $-27 \pm 5.7$ & $-62 \pm 4.9$ & $46 \pm 3.8$ & $1531 \pm 202$ \\
r-SPL & $23 \pm 3.6$ & $-61 \pm 4.4$ & $45 \pm 4.5$ & $1543 \pm 204$ \\
cPrec & $2 \pm 2.8$ & $-51 \pm 3.3$ & $28 \pm 4.8$ & $1522 \pm 330$ \\
dPrec & $-1 \pm 2.9$ & $-69 \pm 3.7$ & $32 \pm 4.7$ & $1579 \pm 188$ \\
PCC & $0 \pm 2.3$ & $-31 \pm 5.6$ & $29 \pm 4.6$ & $1479 \pm 304$ \\
I-LP & $-46 \pm 4.5$ & $-62 \pm 5.5$ & $33 \pm 4$ & $1610 \pm 140$ \\
r-LP & $44 \pm 4$ & $-62 \pm 6.9$ & $31 \pm 4.3$ & $1551 \pm 234$ \\
r-SFG & $19 \pm 4.9$ & $13 \pm 8$ & $55 \pm 4.4$ & $1214 \pm 358$ \\
mPFC & $2 \pm 3$ & $51 \pm 4.7$ & $10 \pm 6.1$ & $1356 \pm 338$ \\
\hline
\end{tabular}

ROI centers and volumes, averaged across participants, $\pm 1 S D . X, Y, Z$ values correspond to Talairach coordinates.

which is why we defined two separate precuneus ROIs (cPrec and $\mathrm{dPrec}$ ) for each participant. This division reflected the previously observed functional and anatomical differentiation in precuneus (Immordino-Yang et al., 2009; Margulies et al., 2009). LP was defined separately in each hemisphere. rSFG was identified only in the right hemisphere. The other intrinsic ROIs were medial, and in close anatomical proximity in the two hemispheres; consequently, we picked a single ROI center and defined a single ROI around that center location that included voxels from both hemispheres.

\section{INTER-AREA CORRELATIONS}

The main analysis computed inter-area correlations (also called "functional connectivity") between the time-series of brain activity in different ROIs (Friston et al., 1996). We extracted the time-series of each voxel in each ROI and averaged across voxels to give an average time-series for each ROI, each participant, and each condition (fixation, NDA, FA). Each such ROI time-series was then $z$-scored by subtracting its mean and dividing by its variance. We obtained the grand average time-series for the extrinsic and intrinsic systems by averaging the $z$-scored time-series of all ROIs belonging to each of the two systems. We then computed the correlation between those two grand average time-series as an overall measure of the level of anti-correlation between the two systems for each of the three conditions (Figures 3 and 4A).

In addition, we computed the inter-area correlation separately for each pair of ROIs, and averaged the Fisher-transformed correlation values across pairs, and across participants. This was done separately for each pair of extrinsic ROIs, each pair of intrinsic ROIs, and for each extrinsic-intrinsic pair (Figure 4B).

Statistical analyses tested for differences in inter-area correlations between conditions (e.g., NDA vs. fixation) and across participants. The correlations were computed separately for each individual participant and for each condition (fixation, NDA, FA), using individually defined ROIs. Paired $t$-tests were then used to assess differences in the correlation values between

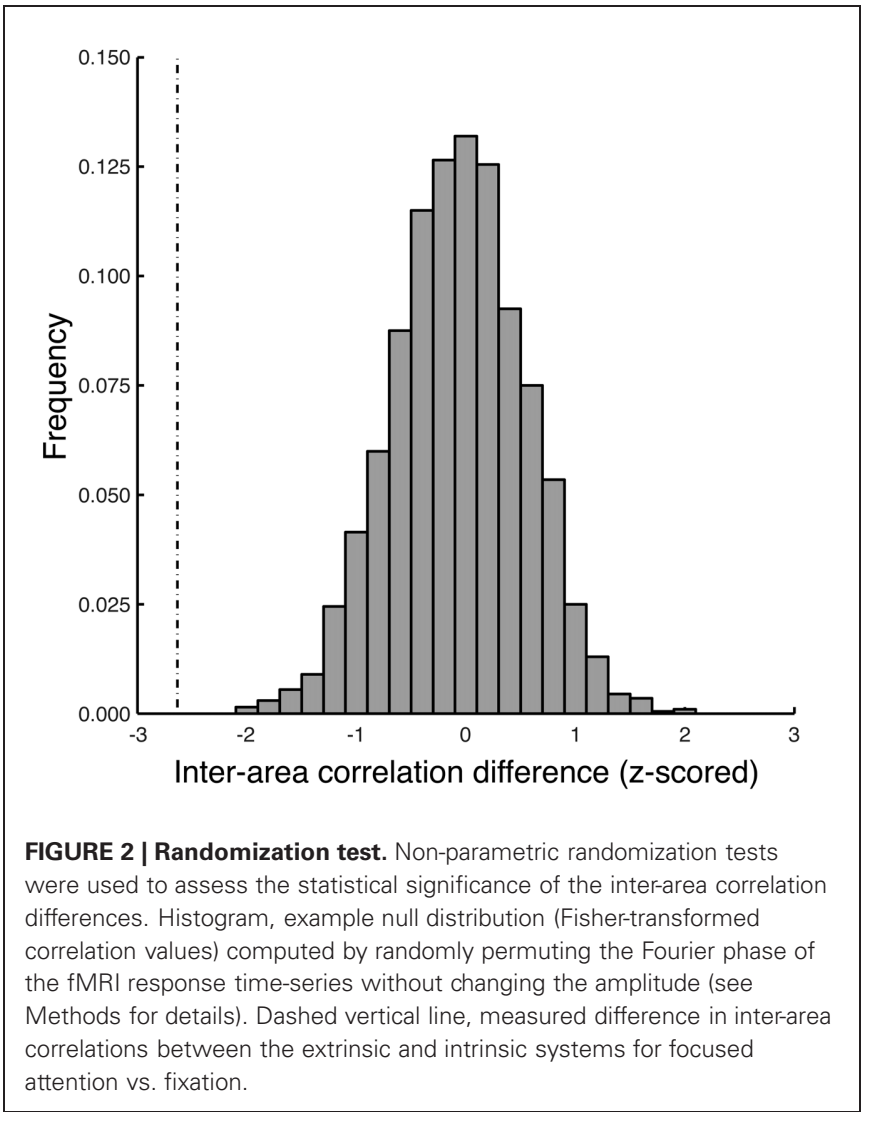

conditions (e.g., NDA vs. fixation). Because correlations are not normally distributed, the $t$-tests were computed on Fishertransformed correlation values.

In addition, to rule out the influence of temporal autocorrelation on the time-series, which might have inflated the correlation statistic, particularly as a smoothing kernel of four TRs had been applied, we also used non-parametric randomization tests to assess the statistical significance of the inter-area correlation differences (Hasson et al., 2008; Lerner et al., 2011; Wang et al., 2010). Specifically, we took the discrete Fourier transform of the time-series in each ROI, randomly permuted its Fourier phase without changing the amplitude, inverted the Fourier transform, and recomputed the correlation between the resulting time-series. This was done for each condition (fixation, NDA, FA), and we computed the differences in the resulting correlation values between conditions (e.g., NDA vs. fixation). This procedure was repeated 1000 times to yield a null distribution for the correlation-differences. Finally, we determined a $p$-value as the fraction of the null distribution (regardless of sign so as to be a two-tailed test) that was as large or larger than the correlation-difference observed without randomization (Figure 2).

\section{VARIANCE OF RESPONSE TIME-SERIES}

We computed the standard deviation of the fMRI response timeseries in each ROI to determine if there was any difference in the amplitude of modulation of the fMRI responses between 
conditions. If there was any such a difference, it might reflect differences in the neural activity between conditions or it might reflect confounding factors such as differences in eye movements, head movements, pulse, or respiration. Specifically, we extracted the time-series of each voxel in each ROI and averaged across voxels to give an average time-series for each ROI, each participant, and each condition (fixation, NDA, FA). Each such time-series was divided by its mean to convert from arbitrary units to percent modulation. We averaged the time-series across voxels for each ROI and computed the standard deviation of the resulting mean time-series. Finally, these standard deviations were then averaged across ROIs and across participants, separately for each condition (fixation, NDA, FA), and separately for the intrinsic ROIs and the extrinsic ROIs (Figure 5).

We used non-parametric randomization tests to assess the statistical significance of any differences in the standard deviations. Specifically, we randomly permuted the condition labels (fixation, NDA, FA) for each ROI separately, and we recomputed the standard deviations. This procedure was repeated 1000 times to yield null distributions for the standard deviations. Finally, we determined a $p$-value as the fraction of the null distribution (regardless of sign so as to be a two-tailed test) that was as large or larger than the observed value without randomization.

\section{RESULTS}

\section{INTER-AREA CORRELATION BETWEEN EXTRINSIC AND INTRINSIC SYSTEMS}

The inter-area correlation between the extrinsic and intrinsic systems depended on whether participants fixated, or engaged in NDA or FA meditations (Figures 3 and 4). We computed the grand-averaged time-series for all voxels in the extrinsic and intrinsic ROIs (see Figure 1 and Methods for details), and then computed the correlation between those two grand average time-series as an overall measure of the level of anticorrelation between the two systems (see Figure 3 for an individual participant example and Figure 4A for the correlations from all participants). Brain activity was significantly more anticorrelated during FA than fixation, and significantly less anticorrelated during NDA than fixation $(p<0.001$, paired $t$-tests; $p<0.005$, phase-randomization test, see Figure 2 and Methods for details).

These differences in anti-correlation were specific to interactions between the extrinsic and intrinsic systems, as there was no evidence for differences in correlations between pairs of intrinsic ROIs nor between pairs of extrinsic ROIs (Figure 4B and Table 2). We computed the inter-area correlation separately for each pair of ROIs. This was done separately for each pair of extrinsic ROIs, each pair of intrinsic ROIs, and for each extrinsic-intrinsic pair. We then averaged the correlation values across pairs of ROIs, and across participants (see Methods for details). Averaged pairwise correlations for extrinsic-intrinsic pairs were negative and differed significantly between conditions. For pairs of extrinsic system ROIs, on the other hand, the correlations were positive and did not significantly differ between conditions. The correlations were also positive for pairs of intrinsic ROIs; there was a marginally significant difference between FA and NDA but not between the other pairs of conditions.

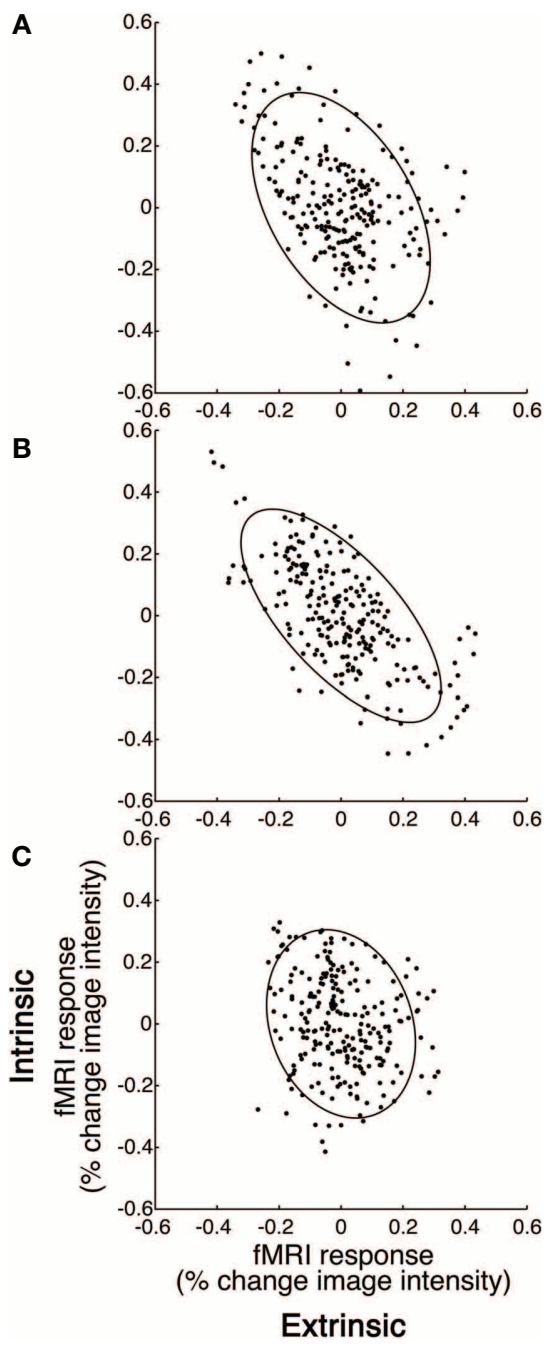

FIGURE 3 | Inter-area correlation. (A) Fixation. (B) Focused attention (FA). (C) Non-dual awareness (NDA). Abscissa, activity in extrinsic system. Ordinate, intrinsic system. Each data point represents the fMRI responses at one time point, averaged across all voxels in each of the two systems, for a typical example participant. Ellipse, level contour of best-fit bivariate normal distribution. The leftward tilt of each ellipse indicates the degree of anti-correlation, although statistics were computed using randomization tests so as not to assume that the $\mathrm{fMRI}$ responses were normally distributed and statistically independent at successive time points (see Methods for details)

\section{VARIANCE OF RESPONSE TIME-SERIES}

There was no evidence for any differences between conditions in the modulation of brain activity, neither in the extrinsic system ROIs nor in the intrinsic ROIs (Figure 5). Because correlation depends on both covariance and variance, the observed differences in the correlations (see above) might have been due to changes in covariance or variance or both. We computed the standard deviations of fMRI response time-series for each ROI in each participant and each condition, then averaged them across ROIs separately for extrinsic ROIs and intrinsic ROIs, and separately for each of the three conditions (fixation, FA, NDA) (see Methods for details). Standard deviations were higher in the intrinsic network 

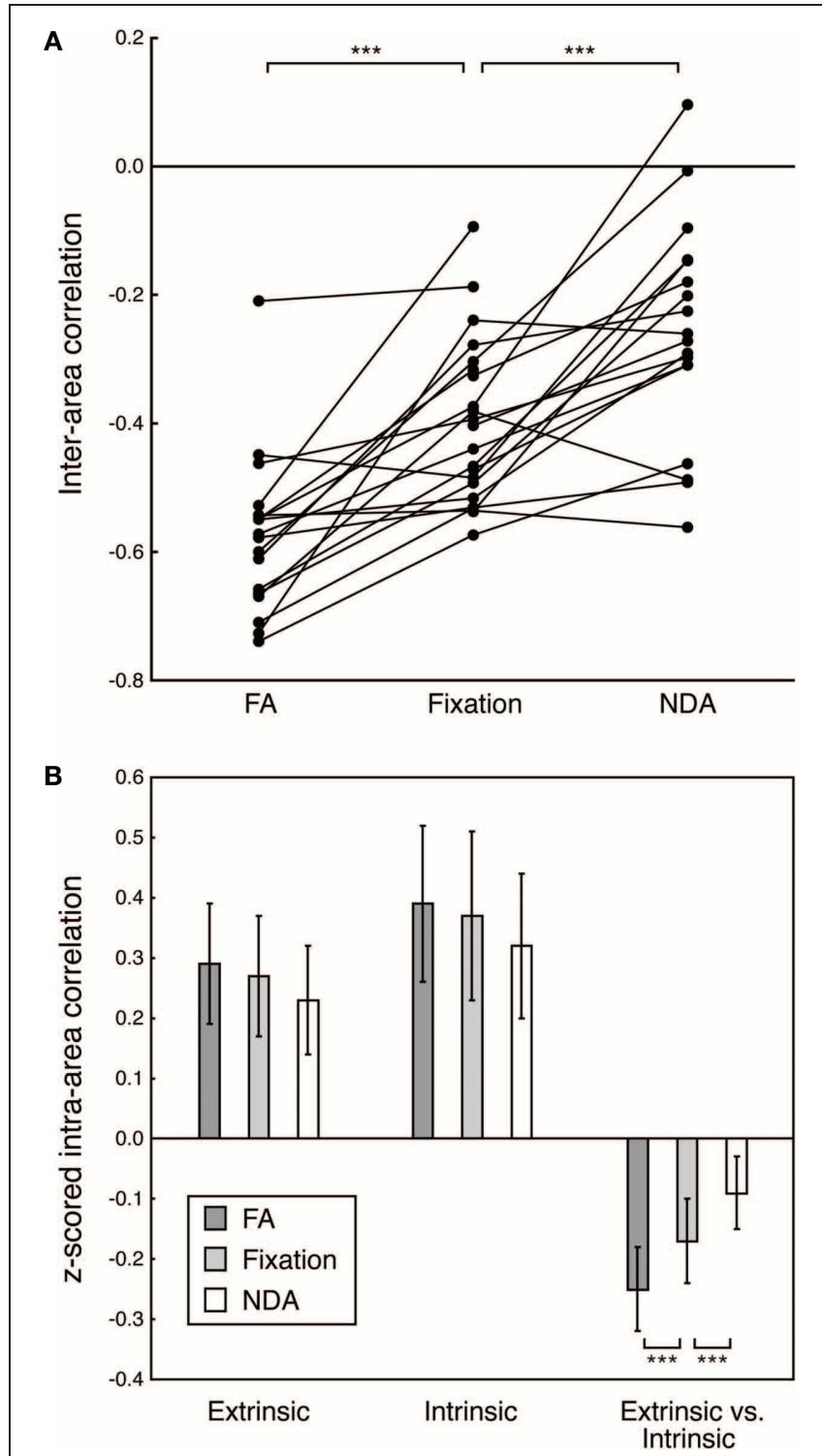

FIGURE 4 | Anti-correlation between extrinsic and intrinsic systems. (A) Correlation between activity in extrinsic and intrinsic systems. FA, focused attention. Fixation, fixation without meditation. NDA, non-dual awareness. Each data point corresponds to fMRI responses from one participant, averaged across all voxels in each of the two systems (extrinsic, intrinsic). Lines connect correlations from the same participant across conditions. Asterisks, statistically significant differences in correlation across participants $(p<0.005$, randomization test). Mean correlations across participants: FA, $r=-0.58$; Fixation, $r=-0.41$; NDA, $r=-0.22$. (B) Correlations between activity in extrinsic and intrinsic ROls. fMRI response time-series were $z$-scored and averaged across voxels in each ROI. A correlation coefficient was then computed for each pair of ROIs, separately for each participant. Height of each bar, Fisher-transformed correlation coefficients, averaged across pairs of ROIs and averaged across participants. Extrinsic, average across all pairs of extrinsic system ROls. Intrinsic, all pairs of intrinsic ROIs. Extrinsic vs. Intrinsic, all extrinsic-intrinsic pairs. Error bars, SEM across participants. Asterisks, statistically significant differences in correlation across participants $(p<0.005$, randomization test).
Table 2 | Statistical significance of average pairwise correlations.

\begin{tabular}{llll}
\hline & fixation vs. FA & fixation vs. NDA & FA vs. NDA \\
\hline Extrinsic & $p=0.8414$ & $p=0.3249$ & $p=0.0536$ \\
& $p=0.6520$ & $p=0.1243$ & $p=0.0879$ \\
Intrinsic & $p=0.7429$ & $p=0.2949$ & $p=0.0699$ \\
& $p=0.5201$ & $p=0.1673$ & $p<0.05$ \\
Extrinsic vs. & $p \approx 0$ & $p \approx 0$ & $p \approx 0$ \\
Intrinsic & $p<0.005$ & $p \approx 0$ & $p \approx 0$ \\
\hline
\end{tabular}

Top number in each cell corresponds to paired t-test. Bottom number in each cell corresponds to phase-randomization test (see Figure 2 and Methods for details).

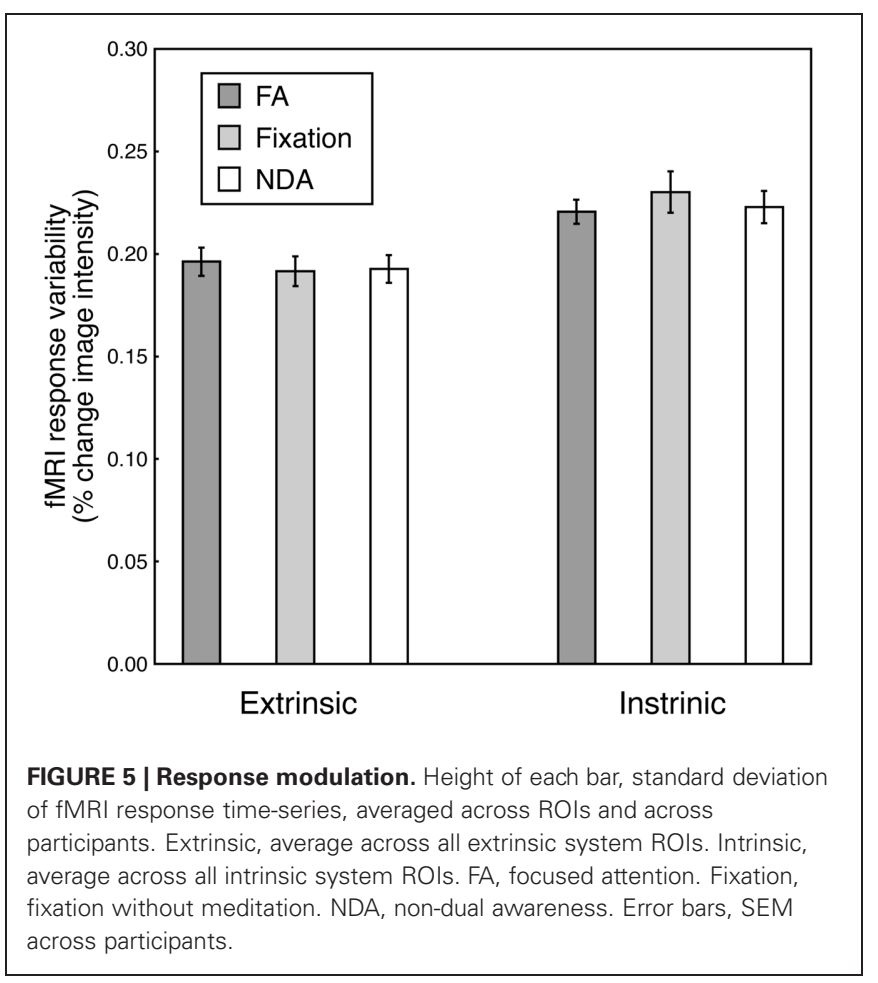

than in the extrinsic one, across all three conditions $(p<0.005$, randomization test). However, the standard deviations were not significantly different across the three conditions (fixation vs. FA: $p=0.9880$ extrinsic, $p=0.4560$ intrinsic, randomization test; fixation vs. NDA: $p=0.4540$ extrinsic, $p=0.2920$ intrinsic, randomization test; FA vs. NDA: $p=0.7440$ extrinsic, $p=$ 0.3580 intrinsic, randomization test). Hence, the differences in the correlations between conditions reflected differences in the interactions between extrinsic and intrinsic system ROIs, not in the modulation of activity within these brain areas.

\section{VARIANCE OF GLOBAL COMPONENT}

We wanted to ensure that the observed differences in anticorrelation between extrinsic and intrinsic systems across meditation styles were not due to differences in the variance of the global component that had been regressed out. If the global component had large amplitude of modulation during one condition 
than for another, it might have artificially generated stronger anti-correlations. We found, however, that there was no difference in the variance of the global components across conditions $(p>$ 0.25 , two-tailed $t$-test and randomization test). Furthermore, the variance of the global component was largest (though not statistically significantly larger) in the NDA condition where anti-correlation between extrinsic and intrinsic was weakest, suggesting that global component variability did not contribute to the anti-correlations.

\section{DISCUSSION}

Our results suggest that practicing different forms of meditation can alter the gross functional organization of the cortex in profoundly different ways. Experienced Tibetan Buddhist meditators exhibited stronger anti-correlation between extrinsic and intrinsic systems during FA meditation than during fixation with no mediation, replicating previous reports. In contrast, the anticorrelations between the two systems were smaller during NDA meditation.

The proposal that the cortex is divided into two broad systems (Gusnard and Raichle, 2001; Fox et al., 2005; Fransson, 2006; Fox and Raichle, 2007; Golland et al., 2007, 2008; Tian et al., 2007; Buckner et al., 2008; Boly et al., 2009; Soddu et al., 2009; Vanhaudenhuyse et al., 2010b), which are antagonistic and compete with one another has generated a great amount of interest over the past several years. According to this proposal, sensory, attention, and motor areas of the brain compose an extrinsic system that is mostly engaged in interpreting external stimuli and in planning and executing appropriate behavioral responses. In contrast a set of far less studied areas, which include the precuneus, the inferior parietal lobule, and the mPFC, are consistently inhibited during externally related tasks and, instead, respond when subjects are asked to imagine their future or contemplate about their current situation or past experiences. The brain areas belonging to the extrinsic and intrinsic systems also exhibit anti-correlation during rest, sleep, and while under anesthesia, when no obvious external or internal task is being performed. These findings have suggested that the competitive and antagonistic nature of neural activity in these two systems represents a fundamental gross organizational principle of the cortex. How flexible this principle is and under what circumstances it can be modulated has been a focus of intense interest and debate. The specificity of our results for the type of meditation strongly supports the conclusion that the anti-correlation between the activity of the extrinsic and intrinsic systems can be modulated in either direction through the choice of a cognitive strategy. Our results may also offer novel evidence in support of current theories regarding the competitive functional roles of extrinsic and intrinsic brain areas. It is tempting to speculate that practicing a meditation that either focuses on, or monitors, external or internal experience (FA and OM), actively increases the competition between extrinsic and intrinsic systems, while practicing a meditation that cultivates awareness in which external and internal experience is integrated (NDA) decreases this competition.

Most relevant to the current study are previous examinations of meditation effects on the correlation within and between the intrinsic and extrinsic systems. All previous studies on the topic have utilized two general styles of meditation: FA and OM. It has been proposed that FA meditations rely primarily on the voluntary endogenous attention, mediated by the dorsal attention network (part of the extrinsic system), while OM style meditations rely primarily on for involuntary exogenous attention/vigilance, mediated by the right-lateralized ventral attention network (Fox et al., 2006; Jha et al., 2007; Corbetta et al., 2008; Austin, 2009; Raffone and Srinivasan, 2010). Consistent with our results, some studies reported that anti-correlations between some areas of intrinsic and extrinsic systems were stronger during FA and OM meditations than without meditation (Farb et al., 2007; Brewer et al., 2011; Kilpatrick et al., 2011). Brewer et al. (2011) also reported that the correlation between areas involved in cognitive control (ACC, dorsolateral prefrontal cortex) and the PCC area in the intrinsic system were higher for experienced meditators than controls, both at rest and during meditation. Also consistent with our results, one previous study reported no evidence for differences in inter-area correlations within each system (i.e., no differences in correlations between areas belonging to the extrinsic system nor between areas belonging to the intrinsic system) (Manna et al., 2010). However, that same study reported no evidence for a difference in inter-area correlations between the two systems when comparing brain activity during FA and OM meditations with brain activity when participants were resting with their eyes closed (Manna et al., 2010), inconsistent with our results. And other studies reported that inter-area correlations within each system were larger during FA and OM meditations than without meditation (Kilpatrick et al., 2011), also inconsistent with our results. Our results, therefore, replicate and extend some of the previous reports, but contradict other findings.

Our results lend further evidence to the claims that NDA meditations are different from both FA and OM meditations. While FA and OM meditations are traditionally regarded as "constructed" states created through deployment of specific attentional strategies, NDA meditations are thought not to involve intentional effort, but to be based on identifying a reflexive awareness that is regarded as "unconstructed" (Mipam and Hopkins, 2006). Additionally, both FA and OM meditations are contentoriented, concerned with the specifics of experience, while NDA meditations could be seen as primarily context-oriented, concerned with the context of experience. Our results showing less anti-correlation between the extrinsic and intrinsic systems during NDA could be interpreted as indicating that NDA may be mediated by neural mechanisms different from the attentional systems mediating FA and OM, or alternatively, that the two attentional systems function differently in NDA, than in FA and OM.

The main novelty of the current study is in reporting that NDA meditation has an opposite effect, to that of FA meditation, on the anti-correlation between intrinsic and extrinsic systems. There are no previous reports of inter-area correlations during NDA meditation. While the anti-correlation has repeatedly been reported to be stronger for FA meditation compared to no meditation, we found that the anti-correlation was smaller for NDA meditation. NDA meditation is different from 
FA and OM meditations in that it enables an atypical state of mind in which extrinsic and intrinsic experiences are increasingly synergistic rather than competing. Our results, therefore, add to the growing body of scientific evidence that mental states induced by different mediation practices correspond to measurably different states of brain activity, and that the typical anti-correlation between the extrinsic and intrinsic systems might reflect the duality of extrinsic and intrinsic experiences.

It is important to consider the pre-processing of the data when interpreting the anti-correlation between intrinsic and extrinsic systems. Specifically, a common step in pre-processing "restingstate" fMRI data (i.e., fMRI measurements in which there is no task or no change in the stimulus/task) is to remove a "global" component, typically the mean response time-course averaged across all gray matter voxels (see Methods). Doing so removes responses that are common across the entire cortex that they may be due to changes in breathing, heart-rate, blood-pressure, and head movements, rather than changes in neural activity. There is some debate in the field regarding the implications of removing this global component and the possibility that doing so might generate an artificial anti-correlation between intrinsic and extrinsic systems (Fox et al., 2009). Regardless, in the current study we performed identical pre-processing steps for all three conditions (FA, NDA, fixation), meaning the observed changes in anti-correlation between intrinsic and extrinsic systems were not dependent of this issue pre-processing step. And there was no evidence for a difference across conditions in the amplitude of modulation of the global component.

Perhaps the biggest challenge in studying the effects of meditation on cortical function is that it is very difficult to objectively determine how and to what extent each participant is meditating (Varela, 1996; Lutz and Thompson, 2003). Are the same precise cognitive strategies being used? Are subjects able to achieve similar levels of practice in the fMRI scanner? There is indeed very limited control over the participants' behavior in this type of experiments, which can lead to a troubling heterogeneity of results across studies. For these reasons, and the following previous studies (Lutz et al., 2004; Brefczynski-Lewis et al., 2007), we recruited participants with many years of experience in Tibetan Buddhist meditation, to maximize expertise and minimize individual differences.

\section{FUTURE DIRECTIONS}

A follow-up study would test naïve participants, provided with a minimal training, to determine if our results are the effect of longterm learning/practice, or if similar results could be produces in a shorter time as well.

\section{ACKNOWLEDGMENTS}

We are grateful to G. Brouwer, U. Hasson, R. Malach, and D. Stanley for assistance with the experiment and for helpful discussions. Supported by the Baumann Foundation, the Mind Science Foundation, and the NYU Center for Brain Imaging.

\section{REFERENCES}

Andrews-Hanna, J. R., Reidler, J. S., Sepulcre, J., Poulin, R., and Buckner, R. (2010). Functional-anatomic fractionation of the brain's default network. Neuron 65, 550-562.

Austin, J. H. (2009). Selfless Insight. Cambridge, MA: MIT Press.

Boly, M., Tshibanda, L., Vanhaudenhuyse, A., Noirhomme, Q., Schnakers, C., Ledoux, D., Boveroux, P., Garweg, C., Lambermont, B., Phillips, C., Luxen, A., Moonen, G., Bassetti, C., Maquet, P., and Laureys, S. (2009). Functional connectivity in the default network during resting state is preserved in a vegetative but not in a brain dead patient. Hum. Brain Mapp. 30, 2393-2400.

Boynton, G. M., Engel, S. A., Glover, G. H., and Heeger, D. J. (1996). Linear systems analysis of functional magnetic resonance imaging in human V1. J. Neurosci. 16, 4207-4221.

Brefczynski-Lewis, J. A., Lutz, A., Schaefer, H. S., Levinson, D. B., and Davidson, R. J. (2007). Neural correlates of attentional expertise in long-term meditation practitioners. Proc. Natl. Acad. Sci. U.S.A. 104, 11483-11488

Brewer, J. A., Worhunsky, P. D., Gray, J. R., Tang, Y. Y., Weber, J., and Kober,
H. (2011). Meditation experience is associated with default mode network activity and connectivity. Proc. Natl. Acad. Sci. U.S.A. 108, 20254-20259.

Buckner, R. L., Andrews-Hanna, J. R., and Schacter, D. L. (2008). The brain's default network: anatomy, function, and relevance to disease. Ann. N. Y. Acad. Sci. 1124, 1-38.

Cahn, B. R., and Polich, J. (2006). Meditation states and traits: EEG, ERP and neuroimaging studies. Psychol. Bull. 132, 180-211.

Castellanos, F. X., Margulies, D. S., Kelly, C., Uddin, L. Q., Ghaffari, M., Kirsch, A., Shaw, D., Shehzad, Z., Di Martino, A., Biswal, B., SonugaBarke, E. J., Rotrosen, J., Adler, L. A., and Milham, M. P. (2008). Cingulate-precuneus interactions: a new locus of dysfunction in adult attention-deficit/hyperactivity disorder. Biol. Psychiatry 63, 332-337.

Conze, E. (1993). A Short History of Buddhism. Grantham, UK: Oneworld.

Corbetta, M., Patel, G., and Shulman, G. L. (2008). The reorienting system of the human brain: from environment to theory of mind. Neuron 58 , 306-324.
Damoiseaux, J. S., Rombouts, S. A., Barkhof, F., Scheltens, P., Stam, C. J., Smith, S. M., and Beckmann, C. F. (2006). Consistent resting-state networks across healthy subjects. Proc. Natl. Acad. Sci. U.S.A. 103 13848-13853.

Demertzi, A., Soddu, A., Faymonville, M. E., Bahri, M. A., Gosseries, O., Vanhaudenhuyse, A., Phillips, C., Maquet, P., Noirhomme, Q., Luxen, A., and Laureys, S. (2011). Hypnotic modulation of resting state fMRI default mode and extrinsic network connectivity. Prog. Brain Res. 19, 309-322.

Farb, N. A. S., Segal, Z. V., Mayberg, H., Bean, J., McKeon, D., Zainab, F., and Anderson, A. K. (2007). Attending to the present: mindfulness meditation reveals distinct neural modes of self-reference. Soc. Cogn. Affect. Neurosci. 2, 313-322.

Fox, M. D., Corbetta, M., Snyder, A. Z., Vincent, J. L., and Raichle, M. A. (2006). Spontaneous neuronal activity distinguishes human dorsal and ventral attention systems. Proc. Natl. Acad. Sci. U.S.A. 103, 10046-10051.

Fox, M. D., and Raichle, M. E. (2007). Spontaneous fluctuations in brain activity observed with functional magnetic resonance imaging. Nat. Rev. Neurosci. 8, 700-711.

Fox, M. D., Snyder, A. Z., Vincent, J. L. Corbetta, M., Van Essen, D. C., and Raichle, M. E. (2005). The human brain is intrinsically organized into dynamic, anticorrelated functional networks. Proc. Natl. Acad. Sci. U.S.A. 102, 9673-9678.

Fox, M. D., Zhang, D., Snyder, A. Z., and Raichle, M. E. (2009). The global signal and observed anticorrelated resting state brain networks. J. Neurophysiol. 101, 3270-3283.

Fransson, P. (2006). How default is the default mode of brain function? Further evidence from intrinsic BOLD signal fluctuations. Neuropsychologia 44, 2836-2845.

Friston, K. J. (1995). Functional and effective connectivity in neuroimaging: a synthesis. Hum. Brain Mapp. 2, 56-78.

Friston, K. J., Frith, C. D., Fletcher, P., Liddle, P. F., and Frackowiak, R. S. (1996). Functional topography: multidimensional scaling and functional connectivity in the brain. Cereb. Cortex 6, 156-164.

Friston, K. J., Jezzard, P., and Turner, R. (1994). Analysis of functional MRI time-series. Hum. Brain Mapp. 1, 153-171. 
Fukunaga, M., Horovitz, S. G., van Gelderen, P., de Zwart, J. A., and Jasma, J. M. (2006). Largeamplitude, spatially correlated fluctuations in BOLD fMRI signals during extended rest and early sleep stages. Magn. Reson. Imaging 24, 979-992.

Gerlach, K. D., Spreng, R. N., Gilmore, A. W., and Schacter, D. L. (2011). Solving future problems: default network and executive activity associated with goal-directed mental simulations. Neuroimage 55, 1816-1824.

Glover, G. H. (1999). Deconvolution of impulse response in eventrelated BOLD fMRI. Neuroimage 9, 416-429.

Goebel, R., Esposito, F., and Formisano, E. (2006). Analysis of functional image analysis contest (FIAC) data with Brainvoyager QX: from singlesubject to cortically aligned group general linear model analysis and self-organizing group independent component analysis. Hum. Brain Mapp. 27, 392-401.

Golland, Y., Bentin, S., Gelbard, H., Benjamini, Y., Heller, R., Nir, Y., Hasson, U., and Malach, R. (2007). Extrinsic and intrinsic systems in the posterior cortex of the human brain revealed during natural sensory stimulation. Cereb. Cortex 4, 766-777.

Golland, Y., Golland, P., Bentin, S., and Malach, R. (2008). Data-driven clustering reveals a fundamental subdivision of the human cortex into two global systems. Neuropsychologia 46, 540-553.

Goldberg, I., Harel, M., and Malach, R. (2006). When the brain loses its self: prefrontal inactivation during sensorimotor processing. Neuron 50, 329-339.

Gusnard, D. A., and Raichle, M. E. (2001). Searching for a baseline: functional imaging and the resting human brain. Nat. Rev. Neurosci. 2, 685-694.

Immordino-Yang, M. H., McColl, A., Damasio, H., and Damasio, A. (2009). Neural correlates of admiration and compassion. Proc. Natl. Acad. Sci. U.S.A. 106, 8021-8026.

Hasson, U., Harel, M., Levy, I., and Malach, R. (2003). Large-scale mirror-symmetry organization of human occipitotemporal object areas. Neuron 37, 1027-1041.

Hasson, U., Malach, R., and Heeger, D. J. (2010). Reliability of cortical activity during natural stimulation. Trends Cogn. Sci. 14, 40-48.

Hasson, U., Yang, E., Vallines, I., Heeger, D. J., and Rubin, N. (2008). A hierarchy of temporal receptive windows in human cortex. $J$. Neurosci. 28, 2539-2550.

Horovitz, S. G., Braun, A. R., Carr, W. S., Picchioni, D., Balkin, T. J., Fukunaga, M., and Duyn, J. H. (2009). Decoupling of the brain's default mode network during deep sleep. Proc. Natl. Acad. Sci. U.S.A. 106, 11376-11381.

Jang, J. H., Jung, W. H., Kang, D. H., Byun, M. S., Kwon, S. J., Choi, C. H., and Kwon, J. S. (2011). Increased default mode network connectivity associated with meditation. Neurosci. Lett. 487, 358-362.

Jha, A., Klein, R., Krompinger, J., and Baime, M. (2007). Mindfulness training modifies subsystems of attention. Cogn. Affect. Behav. Neurosci. 7, 109-119.

Josipovic, Z. (2010). Duality and nonduality in meditation research. Conscious. Cogn. 19, 1119-1121.

Kennedy, D. P., and Courchesne, E. (2008). Functional abnormalities of the default network during selfand other-reflection in autism. Soc. Cogn. Affect. Neurosci. 3, 177-190.

Kilpatrick, L. A., Suyenobu, B. Y., Smith, S. R., Bueller, J. A., Goodman, T., Creswell, J. D., Tillisch, K., Mayer, E. A., and Naliboff, B. D. (2011). Impact of mindfulness-based stress reduction training on intrinsic brain connectivity. Neuroimage 56, 290-298.

Lerner, Y., Honey, C. J., Silbert, L. J., and Hasson, U. (2011). Topographic mapping of a hierarchy of temporal receptive windows using a narrated story. J. Neurosci. 31, 2906-2915.

Lutz, A., Dunne, J., and Davidson, R. (2007). "Meditation and the neuroscience of consciousness," in The Cambridge Handbook of Consciousness, eds P. D. Zelazo, M. Moscovitch, and E. Thompson (Cambridge, UK: Cambridge University Press), 499-551.

Lutz, A., Greischar, L. L., Rawlings, N. B., Ricard, M., and Davidson, R. J. (2004). Long-term meditators self-induce high-amplitude gamma synchrony during mental practice. Proc. Natl. Acad. Sci. U.S.A. 101, 16369-16373.

Lutz, A., Slagter, H. A., Dunne, J. D., and Davidson, R. J. (2008). Attention regulation and monitoring in meditation. Trends Cogn. Sci. 12, 163-169.

Lutz, A., and Thompson, E. (2003) Neurophenomenology: integrating subjective experience and brain dynamics in the neuroscience of consciousness. J. Conscious. Stud. 10, 31-52.

Manna, A., Raffone, A., Perrucci, M. G., Nardo, D., Ferretti, A., Tartaro, A., Londei, A., Del Gratta, C. Belardinelli, M. O., and Romani, G. L. (2010). Neural correlates of focused attention and cognitive monitoring in meditation. Brain Res. Bull. 82, 46-56

Margulies, D. S., Vincent, J. L., Kelly, C., Lohmann, G., Uddin, L. Q., Biswal, B. B., Villringer, A., Castellanos, F. X., Milham, M. P., and Petrides, M. (2009). Precuneus shares intrinsic functional architecture in humans and monkeys. Proc. Natl. Acad. Sci. U.S.A. 106, 20069-20074

Mipam, G., and Hopkins, J. (2006). Fundamental Mind. Ithaca, NY: Snow Lion.

Nir, Y., Hasson, U., Levy, I., Yeshurun, Y., and Malach, R. (2006). Widespread functional connectivity and fMRI fluctuations in human visual cortex in the absence of visual stimulation. Neuroimage 30, 1313-1324.

Pagnoni, G., Cekic, M., and Guo, Y. (2008). Thinking about notthinking: neural correlates of conceptual processing during zen meditation. PLoS One 3, e3083. doi:10.1371/journal.pone.0003083

Preminger, S., Harmelech, T., and Malach, R. (2011). Stimulus-free thoughts induce differential activation in the human default network. Neuroimage 54, 1692-1702.

Radhakrishnan, S. (1995). The Principal Upanishads. New Delhi, India: HarperCollins.

Raffone, A., and Srinivasan, N. (2010). The exploration of meditation in the neuroscience of attention and consciousness. Cogn. Process. 1, 1-7.

Raichle, M. E., and Snyder, A. Z. (2007) A default mode of brain function: a brief history of an evolving idea. Neuroimage 37, 1083-1090; discussion 1097-1099.

Soddu, A., Boly, M., Nir, Y., Noirhomme, Q., Vanhaudenhuyse, A., Demertzi, A., Arzi, A., Ovadia, S., Stanziano, M., Papa, M., Steven Laureys, S., and Malach, R. (2009). Reaching across the abyss: recent advances in functional magnetic resonance imaging and their potential relevance to disorders of consciousness. Prog. Brain Res. 177, 261-274.

Sonuga-Barke, E. J., and Castellanos, F. X. (2007). Spontaneous attentional fluctuations in impaired states and pathological conditions: a neurobiological hypothesis. Neurosci. Biobehav. Rev. 31, 977-986.

Sorg, C., Riedl, V., Mühlau, M., Calhoun, V. D., Eichele, T., Läer, L., Drzezga, A., Förstl, H., Kurz, A., Zimmer, C., and Wohlschläger, A. M. (2007). Selective changes of resting-state networks in individuals at risk for Alzheimer's disease. Proc. Natl. Acad. Sci. U.S.A. 104, 18760-18765.

Stamatakis, E. A., Adapa, R. M., Absalom, A. R., and Menon, D. K. (2010). Changes in resting neural connectivity during propofol sedation. PLoS One 5, e14224. doi: 10.1371/journal.pone.0014224

Talairach, J., and Tornoux, P. (1988). Co-Planar Stereotaxic Atlas of the Human Brain: 3-Dimensional Proportional System: An Approach to Cerebral Imaging. Stuttgart: Georg Thieme.

Tian, L., Jiang, T., Liu, Y., Yu, C., Wang, K., Zhou, Y., Song, M., and Li, K. (2007). The relationship within and between the extrinsic and intrinsic systems indicated by resting state correlational patterns of sensory cortices. Neuroimage 36, 684-690.

Travis, F., and Shear, J. (2010). Focused attention, open monitoring and automatic self-transcending: categories to organize meditations from Vedic, Buddhist and Chinese traditions. Conscious. Cogn. 19, 1110-1118.

Vanhaudenhuyse, A., Demertzi, A., Schabus, M., Noirhomme, Q., Bredart, S., Boly, M., Phillips, C., Soddu, A., Luxen, A., Moonen, G., and Laureys, S. (2010a). Two distinct neuronal networks mediate the awareness of environment and of self. J. Cogn. Neurosci. 23, 570-578.

Vanhaudenhuyse, A., Noirhomme, Q., Tshibanda, L. J., Bruno, M. A., Boveroux, P., Schnakers, C., Soddu, A., Perlbarg, V., Ledoux, D., Brichant, J. F., Moonen, G., Maquet, P., Greicius, M. D., Laureys, S., and Boly, M. (2010b). Default network connectivity reflects the level of consciousness in non-communicative brain-damaged patients. Brain 133, 161-171.

Varela, F. (1996). Neurophenomenology: a methodological remedy to the hard problem. J. Conscious. Stud. 3, 330-350. 
Vincent, J. L., Kahn, I., Snyder, A. Z., Raichle, M. E., and Buckner, R. L. (2008). Evidence for a frontoparietal control system revealed by intrinsic functional connectivity. J. Neurophysiol. 100, 3328-3342.

Wang, H., Freeman, J., Merriam, E. P., Hasson, U., and Heeger, D. J. (2010). Temporal scramble disrupts eye movements to naturalistic videos. J. Vis. 10, 528 .

Conflict of Interest Statement: The authors declare that the research was conducted in the absence of any commercial or financial relationships that could be construed as a potential conflict of interest.
Received: 31 October 2011; accepted: 20 December 2011; published online: 03 January 2012.

Citation: Josipovic Z, Dinstein I, Weber J and Heeger DJ (2012) Influence of meditation on anticorrelated networks in the brain. Front. Hum. Neurosci. 5:183. doi: 10.3389/ fnhum.2011.00183
Copyright (C) 2012 Josipovic, Dinstein, Weber and Heeger. This is an openaccess article distributed under the terms of the Creative Commons Attribution Non Commercial License, which permits non-commercial use, distribution, and reproduction in other forums, provided the original authors and source are credited. 